\title{
USO DA ACUPUNTURA NO TRATAMENTO DE BRUXISMO
}

José Lucas Santos ESTEVES ${ }^{1}$

Lorany da Silva LAIA ${ }^{2}$

Mariela Dutra Gontijo de MOURA ${ }^{3}$

Sérgio Ricardo MAGALHÃES ${ }^{4}$

Soraya de Matos Camargo GROSSMANN ${ }^{5}$

Luiz Cláudio MOREIRA JUNIOR ${ }^{6}$

\begin{abstract}
${ }^{1}$ Acadêmico do curso de Odontologia da Universidade Vale do Rio Verde - UninCor, campus Belo Horizonte. santoslucas806@gmail.com

${ }^{2}$ Acadêmico do curso de Odontologia da Universidade Vale do Rio Verde - UninCor, campus Belo Horizonte. loranylaia@hotmail.com

${ }^{3}$ Doutorado em Estomatologia. Docente do curso de Odontologia da Universidade Vale do Rio Verde - UninCor, campus Belo Horizonte. mariela.moura@unincor.edu.br

${ }^{5}$ Doutorado em Engenharia Biomédica. Docente do curso de Odontologia da Universidade Vale do Rio Verde UninCor, campus Belo Horizonte. sergio.magalhaes@unincor.edu.br

${ }^{5}$ Doutorado em Patologia. Docente do curso de Odontologia da Universidade Vale do Rio Verde - UninCor, campus Belo Horizonte. prof.soraya.grossmann@unincor.edu.br

${ }^{6}$ Graduação em Fisioterapia. Pós-graduação em Acupuntura. Diretor Acadêmico do Instituto Superior de Ciências da Saúde, campus Belo Horizonte.diretor.academico@incisaimam.com.br
\end{abstract}

Recebido em: 28/03/2017 - Aprovado em: 10/06/2017 - Disponibilizado em: 01/07/2017

\begin{abstract}
RESUMO:
O objetivo do trabalho é tratar o bruxismo com acupuntura na tentativa de minimizar ou evitar o contato não funcional dos dentes que pode levar ao desequilíbrio fisiopatológico do sistema estomatognático, uma vez que o bruxismo apresenta etiologia multifatorial. Nesse estudo, foram selecionados 10 pacientes com bruxismo, que apresentaram dor nos músculos masséter superficial e temporal e/ou desgastes dentários. Todos foram avaliados na clínica de odontologia da Faculdade de Odontologia da UninCor e encaminhados ao ambulatório para serem submetidos a sessões de acupuntura, com o máximo de 10 sessões de 30 minutos cada. Após o tratamento com acupuntura, 7 pacientes concluíram ao menos 5 sessões, e relataram melhora na dor e na fadiga dos músculos da mastigação, mesmo os pacientes que não sentiam dor relataram bem estar e relaxamento dos músculos masséter superficial e temporal. A acupuntura é uma terapia não invasiva, e oferece resultados satisfatórios na melhora dos sintomas do bruxismo, tais como o alivio da dor e o relaxamento muscular.
\end{abstract}

Palavras-chave: Bruxismo. Acupuntura. Disfunção Temporomandibular. Medicina Tradicional Chinesa. Desgaste Dentário.

\section{USE OF ACUPUNCTURE IN THE TREATMENT OF BRUXISMO}

\begin{abstract}
:
The objective of this study is to treat bruxism with acupuncture in an attempt to minimize or avoid non-functional contact of teeth that may lead to the pathophysiological imbalance of the stomatognathic system, since bruxism has a multifactorial etiology. In this study, 10 patients with bruxism were selected, who presented pain in the superficial and temporal masseter muscles and / or dental wear. All were evaluated at the dentistry clinic of the UninCor School of Dentistry and sent to the clinic to be submitted to acupuncture sessions, with a maximum of 10 sessions of 30 minutes each. After acupuncture treatment, 7 patients completed at least 5 sessions, and reported improvement in pain and fatigue of chewing muscles, even patients who did not feel pain reported well being and relaxation of the superficial and
\end{abstract}


temporal masseter muscles. Acupuncture is a non-invasive therapy, and offers satisfactory results in improving symptoms of bruxism, such as pain relief and muscle relaxation.

Keywords: Bruxism. Acupuncture. Temporomandibular dysfunction. Traditional Chinese medicine. Dental Discharge

\section{INTRODUÇÃO}

O bruxismo é uma parafunção caracterizada pelo contato não funcional dos dentes, que ocorre de forma consciente ou inconsciente e pode se manifestar durante qualquer período do dia ou da noite e se caracteriza pelo ranger ou apertar os dentes. Não se trata de uma doença, porém quando aparece de forma exacerbada leva ao desequilíbrio fisiopatológico do sistema estomatognático (BATISTA, 2014; COSTA, 2013; CUNALI et al., 2012; MACHADO et al., 2011; PEREIRA et al., 2006).

O bruxismo se destaca com alta prevalência na população, sendo que os sinais e sintomas estão presentes em até $86 \%$ da população ocidental (SÁ \& MEJA, 2010). As contrações musculares nos pacientes com bruxismo podem desenvolver forças pesadíssimas atingindo de 150 a $340 \mathrm{Kg}$ de carga durante períodos de atividades e, assim, causar grandes alterações em dentes, periodonto, músculos e ATMs. O resultado dessas forças, que excedem muito as forças musculares presentes nas funções mastigatórias normais, causa desgaste dentário, cefaleias, hiperatividade dos músculos da mastigação, principalmente à noite, por falta de atuação dos mecanismos sensoriais de proteção (MACIEL, 2003; OKESON, 2000).

Clinicamente, o bruxismo apresenta alterações na musculatura mastigatória, que apresenta sintomas como a fadiga e aumento da tensão dos músculos mastigatórios, principalmente os músculos pterigoideos medial, lateral, músculo masséter e temporal. Pacientes com bruxismo podem apresentar mialgia, miosite, presença de zonas desencadeantes de dor, falta de coordenação muscular, atividade muscular assimétrica, aumento da atividade muscular tônica, espasmos, contratura, supercontração e superestiramento prolongados e aumento na atividade elétrica. Dentre todas as sintomatologias, a mais comum é a fadiga, que consiste na incapacidade do músculo resistir durante um longo período de tempo determinado esforço sem apresentar sinais e sintomas de dor e desconforto (MOLINA, 1997; OKESON, 2000; PRIMO et al., 2009).

O bruxismo é considerado como uma das parafuncionalidades mais problemáticas que acometem o sistema estomatognático, apresenta etiologia multifatorial e seu tratamento permanece uma incógnita. As intervenções atuais para o manejo do bruxismo envolvem terapias com placas de relaxamento muscular, medicamentos, 
fisioterapias, orientação e educação do autocuidado de detectar e controlar o hábito parafuncional. As terapias não invasivas são as terapias mais amplamente utilizadas e prescritas e, de acordo com a literatura, tem sido muito bem sucedidas (PEREIRA et al., 2006; PORPORATTI et al., 2015; SÁ \& MEJA, 2010).

Diante da problemática do bruxismo a acupuntura, área de atuação da Medicina Tradicional Chinesa (MTC) surge como um possível alude a tantas incertezas. Isso porque a acupuntura é uma terapia não invasiva que pode ser aplicada no tratamento do bruxismo (Garbelotti, et al. 2016). A acupuntura, nos pacientes portadores de bruxismo, age liberando serotonina, endorfina, e atua no aumento da amplitude dos movimentos mandibulares e consequentemente nas funções orais, diminuindo o estresse muscular e favorecendo o relaxamento muscular com a consequente diminuição da dor muscular comumente associada (PORPORATTI et al., 2015). Assim, a acupuntura deixa de ser considerada uma terapia alternativa e passa a ser uma possibilidade de tratamento para o bruxismo, além de não oferecer efeitos adversos. A acupuntura utiliza pontos energéticos do corpo para obter respostas específicas de relaxamento e bem estar no paciente, por meio de agulhas, sementes ou massagens em pontos específicos, como aquelas utilizadas no Do-In, que atuam liberando mediadores químicos endógenos, como as cefalinas e endorfinas, com ação analgésica, relaxante e anti-inflamatória (VIANNA et al., 2008).

Para a acupuntura, a doença é resultado da interação entre os agentes agressores e a resposta do organismo, comandada pelo sistema nervoso central (SNC). Além disso, esse SNC sofre a influência do corpo como um todo, inclusive se o corpo estiver enfraquecido, em estado depressivo, sofrendo com ansiedade, pode refletir negativamente sobre o SNC. No caso do bruxismo, por exemplo, se o espasmo muscular for demasiado, poderá levar a uma isquemia e a um circulo inflamatório vicioso, gerando a dor crônica (SOUSA et al., 2014). Com relação às limitações do uso da acupuntura discutem-se as variáveis de confusão tais como o uso placebo, apesar disso, a literatura médica fornece dados suficientes e afirma que a acupuntura age por mecanismos fisiológicos independente do efeito placebo. Dessa forma, há evidência suficiente do valor da acupuntura para expandir seu uso e encorajar mais estudos de sua fisiologia e seu potencial clínico (MEIRELLES \& GONÇALO. 2009). Apesar de oferecer resultados satisfatórios em todos os estudos realizados, o tratamento de bruxismo com acupuntura carece de muita pesquisa e principalmente de regulamentação dos órgãos responsáveis por seu uso nos consultórios odontológicos, sendo assim, esse estudo tem como objetivo estabelecer o uso 
da acupuntura no tratamento do bruxismo na tentativa de aliviar a dor e promover $\mathrm{o}$ relaxamento muscular minimizando os efeitos deletérios do bruxismo no sistema estomatognático.

\section{METODOLOGIA}

Foram selecionados dez pacientes da Faculdade de Odontologia da Universidade Vale do Rio Verde (UNINCOR), campus Belo Horizonte no qual todos assinaram o termo de consentimento livre e esclarecido.

Para o preenchimento de ficha clínica e anamnese, foi utilizado um duplo cego, composto de dois alunos de graduação, onde o primeiro aluno fez o preenchimento da ficha clínica e a anamnese, que consistiu na palpação do músculo masséter na sua origem, corpo e inserção e na palpação do músculo temporal na sua região anterior, média e posterior.

Em seguida, o segundo aluno repetiu o mesmo procedimento anteriormente detalhado na presença exclusiva do professor sem que o primeiro aluno presenciasse essa etapa, com o intuito de calibrar a palpação e estabelecer o diagnóstico correto do bruxismo. Para a calibragem da força aplicada durante a palpação dos músculos da mastigação, foi utilizada previamente uma balança para medir a intensidade de peso, cuja força variou entre $500 \mathrm{~g}$ a $1000 \mathrm{~g}$.
A intensidade da dor nos músculos masseter superficial e temporal foi mensurada com a escala visual analógica (EVA) da seguinte forma: cada paciente recebeu orientação para graduar sua dor na escala que apresentava valores de 0 (zero) a 10 (dez), onde zero significa ausência de dor ou desconforto e dez, o máximo de dor experimentada pelo paciente, a intensidade foi avaliada pelo relato do paciente imediatamente após episódios de palpação dos músculos masseter superficial e temporal pelo duplo cego.

Na EVA o paciente teve visualização de caras que expressavam dor, e de acordo com a dor que sentia indicava uma das expressões, onde cada expressão era quantificada por um número que variou de $0 \mathrm{a}$ 10 , sendo que somente o pesquisador teve acesso aos números, podendo assim quantificar a dor sentida pelo paciente sem a interferência do mesmo.

Os critérios de inclusão nessa pesquisa foi o paciente apresentar bruxismo, avaliado a partir da presença de dor à palpação nos músculos masseter e temporal estabelecido através da (EVA); presença de desgaste dental, fratura nos dentes e/ou nas restaurações; apresentar sinais de ranger ou apertar os dentes, existência de dentes achatados, fraturados, lascados ou soltos, exposição das camadas mais profundas como dentina; aumento da sensibilidade dentária; presença de retração gengival; distúrbios do 
sono tais como movimentos corporais amplos, alterações respiratórias (apneia); aumento das atividades musculares; hipertrofia muscular; fadiga e tensão mastigatória; limitações de abertura de boca; dificuldade de realizar movimentos mandibulares ao acordar; cefaleias ocasionais no músculo temporal; dor em músculos mastigatórios em repouso ou durante a função mandibular.

Os critérios de exclusão foram ausência de dor nos músculos mastigatórios masséter superficial e temporal, ausência de desgaste dental; uso de medicamentos ansiolíticos ou antidepressivos; uso de drogas ilícitas como cocaína, maconha, crack ou qualquer outra droga que deprima o SNC; pacientes portadores de válvulas cardíacas; portadores de doença mental, como epilepsia no sonho; uso de aparelhos ortodônticos; pacientes edêntulos ou que apresente extremidades livres.

Logo após a avaliação na clínica odontológica da UNINCOR os pacientes selecionados, foram encaminhados à clínica de acupuntura do Instituto de Ciências da Saúde/ Instituto Mineiro de Acupuntura e Massoterapia (INCISA/ IMAM), onde foram realizadas as sessões de acupuntura que seguiram o protocolo da própria clínica. Como não existe um protocolo clínico padrão para a seleção dos pontos utilizados em cada tratamento, a acupuntura foi aplicada com base em dados biopsicossociais colhidos na anamnese do paciente pela medicina tradicional chinesa (MTC). Segundo a literatura mais recente, os pontos mais comumente empregados nos protocolos para o tratamento de bruxismo e dores orofaciais são:

E6-Proeminência do músculo masseter que relaxa os músculos faciais e melhora o Qi da articulação temporomandibular;

E7-No centro da depressão entre a incisura da mandíbula e a borda inferior do arco zigomático, que melhora as funções da ATM, alivia espasmos e dor;

TA17-No ponto em que o lóbulo da orelha toca o pescoço tem função de relaxar os tendões e músculos (Figura 1);

TA21 - Na cavidade intraóssea antes do trago e acima do côndilo trata dor de artrite temporomandibular e dor mandibular ( Figura $1)$;

ID18-Na margem inferior do arco zigomático, na margem anterior do músculo masseter, alivia a dor, acalma a mente, trata dor na face e espasmos nervo facial (Figura $1)$;

Taiyaing (face) - Entre a extremidade lateral da sobrancelha e o epicanto lateral dos olhos, acalma a dor, trata espasmos musculo facial;

Yintang (cabeça) - Linha media da face entre as sobrancelhas, interrompe a dor, acalma a mente, trata cefaleia;

IG4- Entre os metacarpos I e II, na saliência muscular, trata cefaleias, dores na 
face, bochechas, trismo, anestesia cabeça e pescoço (Figura 2)

E44 - (Neiting) Sala interna. No dorso do pé, na depressão entre o primeiro e segundo metatarso, próximo à margem da prega interdigital (Figura 3), (GARBELOTTI et al., 2016).

Neste estudo os pontos utilizados foram; ID 18, TA 17, TA 21, IG4 e E44, conforme as figuras 1,2 ,e 3 .

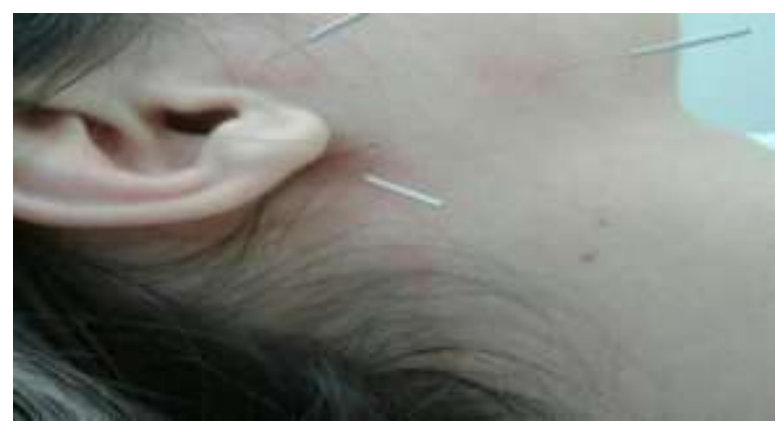

Figura 1: Paciente atendido na acupuntura. Acupontos: ID 18, TA 17, TA 21.

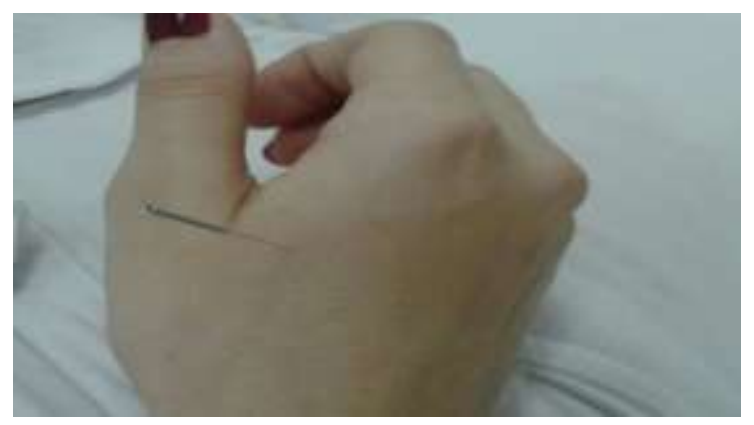

Figura 2: Paciente atendido na acupuntura. Acuponto IG4.



Figura 3: Paciente atendido na acupuntura. Acuponto E44.

A duração do tratamento costuma variar de caso para caso, no entanto para esse estudo teve uma duração de três meses com números de sessões aumentando ou diminuindo dependendo da necessidade de cada paciente. Foi previsto um número máximo 10 sessões para cada paciente com duração de 30 minutos cada.

Após o fim das sessões de acupuntura, os pacientes foram reavaliados na clínica odontológica da faculdade de odontologia da UNINCOR para preenchimento de nova ficha clínica. Nesta reavaliação foi realizado nova ficha clínica com os mesmos requisitos observados previamente no início do tratamento, com o objetivo de comparar a melhora ou não das manifestações clínicas do bruxismo após o tratamento com a acupuntura. Também foi repetida a palpação dos músculos masséter superficial e temporal pelo duplo cego como critério de comparação para estabelecer a eficácia do tratamento do bruxismo com a acupuntura.

Após essa etapa todos os dados foram analisados a fim de oferecer aos pesquisadores e profissionais da odontologia bases e fundamentos para aplicação desta técnica milenar nos consultórios odontológicos, e oferecer aos seus pacientes um tratamento humanizado.

\section{RESULTADOS}

Dos dez pacientes selecionados na clínica de odontologia 80\% (8), são mulheres e $20 \%$ (2) são homens. Do total de pacientes, 
três desistiram de participar dos estudos, dos sete pacientes que continuaram, dois realizaram 10 sessões, um paciente realizou 8 sessões, 2 realizaram 6 sessões e dois pacientes realizaram 5 sessões, atendendo assim o número mínimo de sessões previstas.

Os valores referentes à dor nos músculos masséter (gráfico 1) e temporal (gráfico 2), variou de paciente para paciente e também de músculo para músculo conforme os gráficos previamente listados.

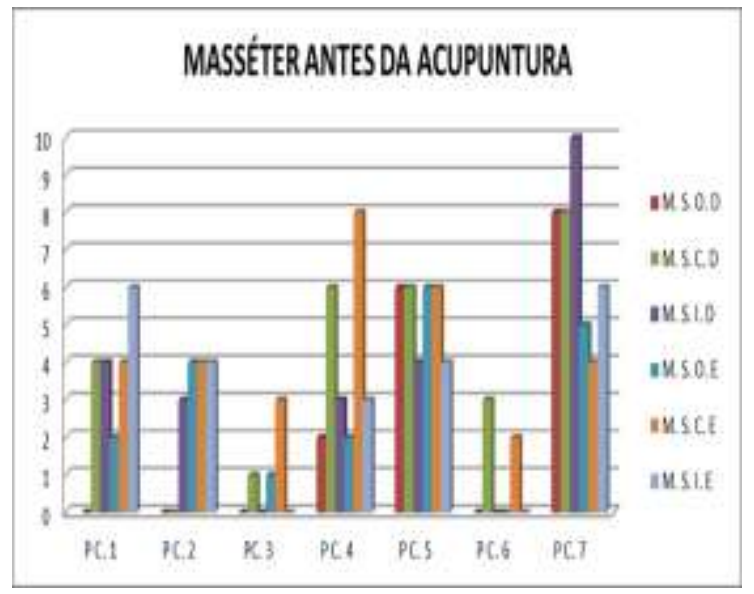

GRÁFIGO 1: M.S.O.D = Masséter superficial origem direito; M. S. C.D = Masséter superficial corpo direito; M.S.I.D = Masséter superficial inserção direito; M. S. O. $\mathrm{E}=$ Masséter superficial origem esquerdo; M. S. C.E = Masséter superficial corpo esquerdo; M.S.I. E = Masséter superficial inserção esquerdo; M. S. I. E = Masséter superficial inserção esquerdo. P C.= Paciente.

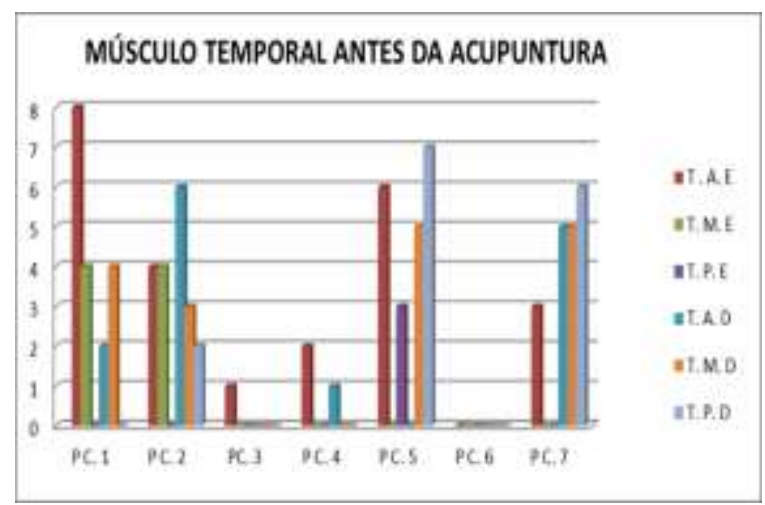

GRÁFICO 2: T. A. E = Temporal anterior esquerdo; T.M.E = Temporal médio esquerdo; T. P. $\mathrm{E}=$ Temporal posterior esquerdo; T. A. D = Temporal anterior direito; T.M.D = Temporal médio direito; T. P.D = Temporal posterior direito; $\mathrm{P} \mathrm{C}=$ Paciente
Os resultados após as seções de acupuntura atenderam as expectativas, uma vez que todos os pacientes apresentaram melhora significativa na dor nos músculos masséter e temporal, conforme se pode observar nos gráficos 3 e 4 , respectivamente.

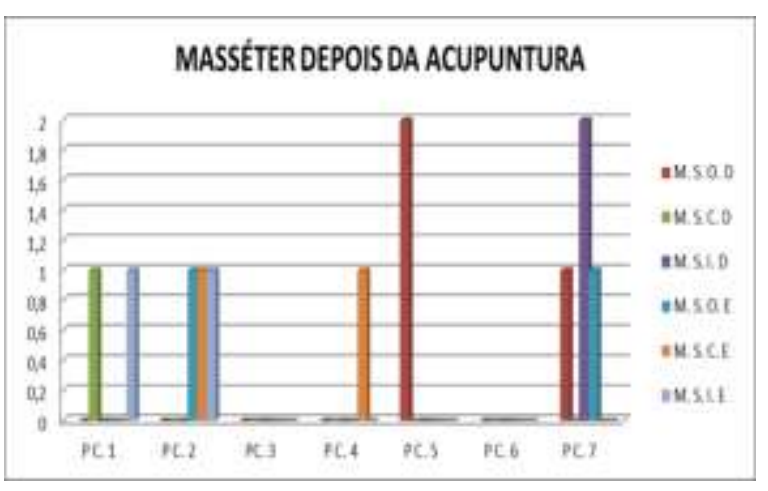

GRÁFIGO 3: M.S.O.D = Masséter superficial origem direito; M. S. C.D = Masséter superficial corpo direito; M.S.I.D = Masséter superficial inserção direito; M. S. O. E = Masséter superficial origem esquerdo; M. S. C.E = Masséter superficial corpo esquerdo; M.S.I. E = Masséter superficial inserção esquerdo; M. S. I. E = Masséter superficial inserção esquerdo. P C.= Paciente

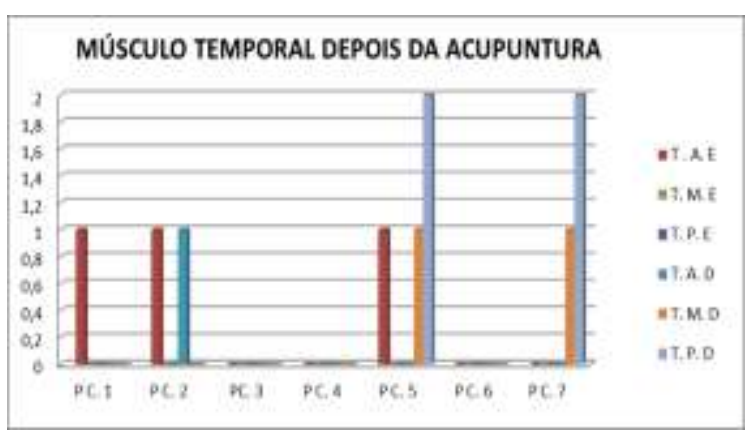

GRÁFICO 4: T. A. E = Temporal anterior esquerdo; T.M.E = Temporal médio esquerdo; T. P. $\mathrm{E}=$ Temporal posterior esquerdo; T. A. D = Temporal anterior direito; T.M.D = Temporal médio direito; T. P.D $=$ Temporal posterior direito; $\mathrm{P} \mathrm{C}=$ Paciente

Todos os pacientes relataram melhorias não somente na dor, mas também no estado emocional, se sentindo mais calmos e relaxados. Dois pacientes ainda relataram melhoras em enfermidades que já eram de conhecimento como gastrite e psoríase, o que comprova a atuação sistêmica da acupuntura. 


\section{DISCUSSÃO}

Este estudo forneceu dados que mostram que a acupuntura é uma técnica indicada e recomendada pela literatura nacional e internacional para tratar $\mathrm{o}$ bruxismo, assim como na maioria dos artigos selecionados e descritos, a acupuntura sozinha ou como terapia complementar ou mesmo em comparação com outras técnicas tem demonstrado ser superior e eficiente para melhorar a dor e a fadiga muscular em pacientes com bruxismo (MORAIS et al., 2012).

A acupuntura atua no combate ao estresse mental, diminui a ansiedade e melhora a qualidade do sono, em uma abordagem holística da acupuntura permite lidar com outras morbidades, bem como com problemas integrativos, como dor de cabeça e dor corporal, visando alcançar o equilíbrio físico e mental, indo assim, de encontro aos resultados obtidos na pesquisa, no qual os pacientes fazem menção a presença de estado de calmaria e tranquilidade após a terapia, com diminuição significativa do estresse (GOMES et al., 2006).

Estudos mostraram que $9,9 \%$ dos americanos usam acupuntura para tratar a enxaqueca ou outras dores de cabeça, os médicos geralmente afirmam que em pouco tempo de tratamento, tais como 12 sessões, durante um período de 3 meses, pode ter um impacto a longo prazo sobre a frequência e a intensidade de episódios de cefaleia e que $50 \%$ dos paciente relataram a diminuição das dores (LINDE et al., 2011) .

\section{SOUZA et al., (2007); MEIRELLES}

\& GONÇALO, (2009) relataramm que pacientes com bruxismo submetidos ao tratamento com acupuntura tiveram redução da dor logo após o tratamento em $71 \%$ dos casos, sendo assim, a experiência de casos clínicos tratados com a acupuntura mostrou resultados promissores com melhoras na escala da dor e principalmente na qualidade de vida, e sugere um protocolo incluindo o bruxismo em um único padrão de tratamento, corroborando assim com os resultados encontrados, no qual $90 \%$ dos pacientes que concluíram as seções relataram melhora na dor e na fadiga do músculo masseter e do músculo temporal.

No entanto MACEDO, (2008) afirmou que a prevalência do bruxismo na população é imprecisa, devido aos estudos que são baseados em populações e metodologias diferentes. Ao se comparar a medicina tradicional chinesa com a medicina ocidental, a grande diferença entre elas está no fato de que na medicina oriental, as emoções são consideradas fatores causais das patologias, logo, não tem como tratar corpo e mente separadamente, assim a abordagem é holística para cada paciente enquanto que, na medicina ocidental, o cérebro está no topo da pirâmide do corpo e da mente, onde as emoções afetam o sistema límbico cerebral e os impulsos 
nervosos acionam o hipotálamo e então atravessam os centros nervosos simpáticos e parassimpáticos do qual então alcançam os órgãos internos. Daí essa emoção que promove o impulso nervoso é então transmitido a um órgão relevante causando o dano manifesto no paciente (MEIRELLES \& GONÇALO, 2009).

Considerando os achados literários e os resultados obtidos na revisão é sugerido que a acupuntura possa continuar a ser uma oportunidade terapêutica em pacientes que sofrem com bruxismo. A questão clinicamente mais relevante não é obviamente se a acupuntura funciona, mas como ela se comporta em relação ao tratamento padrão. Portanto, são necessários estudos com amostras maiores e períodos de observação mais longos para obter um conhecimento mais profundo sobre a eficácia da acupuntura nos pacientes com bruxismo (JUNG et al., 2011).

Estudos realizados por SOUZA et al., (2014), em pacientes com bruxismo, comprovam a redução da dor, bem como a manutenção desta redução após o fim tratamento com acupuntura, e apontam a acupuntura como um excelente método complementar ou convencional nos casos de dores envolvendo músculos como o bruxismo, assim, corroborando com os estudos realizados previamente (JUNG et al., 2011; LINDE et al., 2011).
A literatura é unanime quanto à necessidade de realização de mais ensaios para comprovar a eficácia do uso da acupuntura no tratamento das dores orofaciais e do bruxismo, no entanto estudos mostram que a ação dessa terapia pode estar vinculada ao efeito placebo, devendo então novas pesquisas ser realizadas, de preferência grandes ensaios multicêntricos, para representar de forma válida a variabilidade destas intervenções nos procedimentos de rotina (LINDE et al., 2011). .

Porém há de se questionar o efeito placebo desta terapia uma vez que a mesma pode ser usada em seres desprovidos de capacidade intelectual de raciocínio lógico como animais e plantas, obtendo nesses, resultados também satisfatórios (LINDE et al., 2011).

\section{CONSIDERAÇÕES FINAIS}

Os dados do presente estudo apontam que o tratamento do bruxismo com acupuntura reduziu a dor e promoveu o relaxamento muscular, proporcionou alivio do bruxismo, minimizando seus efeitos deletérios no sistema estomatognatico, bem estar aos pacientes, além de relatos na melhora até em outras áreas não correlatas com bruxismo. Sendo assim pode-se concluir que a acupuntura é de grande benefício, não só para os pacientes portadores de bruxismo, mas 
também para qualquer outro tipo de dor de origem muscular.

\section{REFERÊNCIAS}

BATISTA, Tcherllysie de Andrade. Classificação e etiologia do bruxismo e a importância da mastigação no desenvolvimento do sistema estomatognático em crianças. Universidade Estadual de Londrina 2014. Disponível em: <www.uel.br/graduação>. Acesso em: 18 fev. 2017.

COSTA, Soraia Veloso da. Bruxismo na infância: Estudo de caso clinico aleatório sobre fatores relacionados à ocorrência e influencia na qualidade de vida. 2017. 132 f. Dissertação (Mestrado). Faculdade de Odontologia de Bauru. Universidade de São Paulo, 2013. Disponível em:

<www.teses.usp.br/teses>. Acesso em: $18 \mathrm{fev}$. 2017.

CUNALI, Rafael Schlogel. et al. Bruxismo do sono e disfunções temporomandibulares: revisão sistêmica. Revista Dor, São Paulo, v.13, n.4, p. 360-364, dez. 2012. Disponível em: <http//www.scielo.br/scielo.php>. Acesso em: 18 jan. 2017.

GARBELOTTI, Thânia Orlando. et al.

Effectiveness of acupuncture for temporomandibular disorders and associated symptoms. Revista Dor, São Paulo, v.17, n.3, p. 223-227, set.2016 . Disponível em: $<$ http://www.scielo.br/scielo. phpsc>. Acesso em: 27 fev. 2017.

GARBELOTTI, Thânia Orlando. et al. Eficiência da acupuntura no tratamento das disfunções temporomandibulares e sintomas associados. Revista Dor, São Paulo, v. 17, n. 3, p. 223-227, Sept. 2016. Disponível em: <http://www.scielo.br/scielo. php>. Acesso em: 18 Jan. 2017.
GOMES, Marden Batista. et al. Limiar de dor á pressão em pacientes com cefaleia tensional e disfunção temporomandibular. Ciências Odontológicas UNESP. São Paulo 2006. Disponível em:

<http://ojs.fosjc.unesp.br/index.php>. Acesso em: 27 de fev. de 2017.

JUNG, Aram. et al. Acupuncture for treating temporomandibular joint disorders: a systematic review and metaanalysis of randomized, sham-controlled trials. May 2011Volume 39, Issue 5, Pages 341-350. Disponível em:

<http://bases.bireme.br>. Acesso em: $18 \mathrm{de}$ fev. de 2017.

LINDE, Klaus. Acupuncture for tension-type headache. Cochrane Database Syst Revista Author manuscript. 2011. Disponível em: <http://bases.bireme.br>. Acesso em: $18 \mathrm{de}$ fev. de 2017.

MACEDO, Cristiane Rufino de. Bruxismo do Sono. Revista Dental Press Ortop Facial. Maringá, SP. V. 13, n. 2, p. 18-22. Mar./ abr. 2008. Disponível em: < www.scielo.br/pdf/dpjo/v16n2/a08v16n2>. Acesso em: 27 de Fev. de 2017.

MACHADO, Eduardo. et al. Bruxismo do sono: possibilidades terapêuticas baseado em evidências. Dental Press Journal

Orthodontic. P. 58-64. Março e abril 2011. Disponível em: < www.scielo.br/pdf/dpjo/v16n2/a08v16n2>. Acesso em: 27 de Fev. de 2017.

MACIEl, Roberto N. Oclusão e ATM:

Procedimentos Clínicos. São Paulo: Santos, Com. Imp. Ltda., 1996. 367 p.

MEIRELLES, Maria Paula Maciel Rando; GONÇALO, Camila da Silva. Manejo da dor Orofacial através do tratamento com acupuntura: relato de um caso. Revista Odontológica da Unesp, Araraquara, v. 38, n. 6, p.379-382, 10 dez. 2009. Mensal. Disponível em: 
<www.revodontolunesp.com.br>. Acesso em: 10 fev. 2017.

MOLINA, Omar Franklin. Placas de mordida na terapia oclusal. São Paulo: Pancast. 1997. p. 37-59. Disponível em: <http://bases.bireme.br>. Acesso em: $18 \mathrm{fev}$. 2017.

MORAIS, Yane Marinheiro de. et al. Uso da acupuntura na odontologia: uma revisão literária. In: Congresso Brasileiro Da Saúde, 17, 2012, Florianópolis - Conbracis, 2012. v. 4, p. 13 - 19. Disponível em:

<www.conbracis.com.br >. Acesso em: $18 \mathrm{fev}$. 2017.

OKESON, Jeffrey P. Tratamento das desordens temporomandibulares e oclusão. 4. ed. São Paulo: Artes Médicas, 2000. p.483 $-490$.

PEREIRA, Rafaelle Pessoa Alves. et al. Bruxismo e Qualidade de Vida. Revista Odonto Ciência - Fac. Odonto/PUCRS, v. 21, n. 52, abr./jun. 2006. Disponível em: <www.revistaseletronicas.pucrs.br>. Acesso em: 18 fevereiro de 2017.

PORPORATTI, André Luís. et al. Acupuncture therapeutic protocols for the management of temporomandibular disorders. Revista Dor, São Paulo, v. 16, n. 1, p. 53-59,
Mar. 2015, Available from:< www.scielo.br/scielo.php>. Acesso em: 18 Jan. 2017.

PRIMO, Paula Patrícia. et al. Considerações fisiopatológias sobre bruxismo. Arquivos de Ciências da Saúde da UNIPAR Umuarama, v. 13, n. 3, 2009. Disponível em: <//www.scielo.br/scielo.php>. Acesso em: 18 jan. 2017.

SÁ, Rafaela Oliveira Franco de; MEJA, Dayana Priscila Maia. Uso da acupuntura no tratamento de Disfunção

Temporomandibular.. Faculdade FAIPE. Pernambuco 2015. Disponível em: <http:/scholar.google.com.br/scholar>. Acesso em: 18 fev. 2017.

SOUSA, Maria da Luz Rosário de. et al. Efeito da acupuntura em adultos com disfunção temporomandibular. Revista de Odontologia da Universidade Cidade de São Paulo. 2014. Disponível em:< http://www.scielo.br/scielo.php>. Acesso em: 18 jan. 2017.

VIANNA, R S; Souza. et al. A Acupuntura e sua aplicação na Odontologia 2008; 10(4):4852 UFES Revista Odontologia. Disponível em : < http://www.scielo.br/scielo.php>. Acesso em: 18 jan. 2017. 